\section{(A) Check for updates}

Cite this: Polym. Chem., 2020, 11 5844

Received 18th June 2020,

Accepted 19th August 2020

DOI: $10.1039 / \mathrm{d} 0$ py00878h

rsc.li/polymers

\title{
Epoxy-functionalised 4-vinylguaiacol for the synthesis of bio-based, degradable star polymers via a RAFT/ROCOP strategy $\dagger$
}

\author{
Thomas M. McGuire, ${ }^{a}$ Masato Miyajima, ${ }^{\mathrm{b}}$ Mineto Uchiyama, (D) ${ }^{\mathrm{b}}$ \\ Antoine Buchard (D) *a and Masami Kamigaito (D) *b
}

\begin{abstract}
An epoxy derivative of a naturally occuring vinylphenolic compound, 4-vinylguaiacol (4VGEP), was used for the synthesis of a well-defined $\left(M_{w} / M_{n}=1.08-1.14\right)$, bio-based styrene-type polymer. Block copolymers of 4VGEP with styrene and diethylacrylamide were also prepared and used as macro-monomers in metal, and metal-free, ring-opening copolymerisation (ROCOP) with cis-4-cyclohexene-1,2-dicarboxylic anhydride to form ester cross-linked star polymers in high yields $(82-90 \%)$, with narrow dispersity $\left(M_{w} / M_{n}=\right.$ 1.27-1.40). Finally, the selective degradation of the ester core of the styrene-based star was achieved under acidic conditions.
\end{abstract}

\section{Introduction}

The valorisation of renewable resources for the preparation of degradable polymers is a rapidly developing field in macromolecular chemistry in view of sustainability. ${ }^{1-4}$ In addition to renewability, the characteristic chemical structures of natural products can enhance properties and functionalities of synthetic polymers. More specifically, the oxygen-rich nature of renewable feedstocks can act as a useful chemical handle for stimuli-responsive degradability. ${ }^{5}$

Star polymers are composed of a core attached to three or more linear polymers or arms. ${ }^{6}$ Advances in the controlled synthesis of star polymers have led to their applications being posited in many areas of chemistry, including in drug delivery, ${ }^{7,8}$ drug encapsulation, ${ }^{9}$ gene delivery, ${ }^{10}$ imaging, ${ }^{11-13}$ and catalysis. ${ }^{14,15}$ Reversible-addition-fragmentation chain transfer (RAFT) polymerisation is a highly versatile method for the preparation of functional polymers, including star polymers. ${ }^{16,17}$ Broadly, there are two strategies for the synthesis of star polymers using RAFT polymerisation: arm-first or core-first. In a core-first approach, linear arms can be

\footnotetext{
${ }^{a}$ Centre for Sustainable and Circular Technologies, Department of Chemistry, University of Bath, Claverton Down BA2 7AY, Bath, UK.

E-mail: a.buchard@bath.ac.uk

${ }^{b}$ Department of Molecular and Macromolecular Chemistry, Graduate School of Engineering, Nagoya University, Nagoya 464-8603, Japan.

E-mail: kamigait@chembio.nagoya-u.ac.jp

$\dagger$ Electronic supplementary information (ESI) available: Chemical suppliers, purification of starting materials, instrumentation, synthetic procedures, characterisation of 4 VGEP, ${ }^{1} \mathrm{H}$ and ${ }^{13} \mathrm{C}$ NMR spectra of 4 VGEP and polymers, SEC traces, DSC thermograms and TGA thermograms. See DOI: 10.1039/d0py00878h
}

polymerised from a multi-functional initiating core. Alternatively, when using an arm-first approach, linear polymers are synthesised initially and a core is grafted from the arms through careful choice of polymerisation conditions. Typically, star polymers synthesised using RAFT in an arm-first approach, are formed by cross-linking vinyl-functionalised polymers with multi-vinyl compounds. ${ }^{6,18-21}$ This creates a core linked by $\mathrm{C}-\mathrm{C}$ bonds, the controlled degradation of which is challenging.

Some of us have recently reported the polymerisation of 4-vinylguaiacol (4VG), derived by decarboxylation of naturally occurring ferulic acid. ${ }^{22-25}$ The presence of a hydroxyl group in $4 \mathrm{VG}$ allows for easy functionalisation of $4 \mathrm{VG}$-derived monomers. One potential modification could be through the incorporation of an epoxide moiety, which are versatile functionalities for a number of chemical transformations, including ring opening copolymerisation (ROCOP) with cyclic anhydrides to form polyesters. ${ }^{26}$ Practically, ROCOP can offer advantages over ring opening polymerisation (ROP) of lactones as the properties of the resultant polymer can be tuned through selection of the appropriate co-monomers, thus avoiding lengthy lactone syntheses.

The reactivity of epoxy and vinyl functional groups is generally orthogonal, and has been exploited in one-pot sequential, ${ }^{27,28}$ simultaneous $^{29}$ or switchable ${ }^{30-33}$ block-copolymer syntheses through design of bespoke catalyst/initiator systems. ${ }^{34}$ However, the number of epoxide-functionalised, styrene-type polymers remain relatively small, ${ }^{35-37}$ and we envisaged that epoxy-functionalised 4VG (4VGEP) could act as a bifunctional monomer, which may be suitable for both RAFT and ROCOP, and useful for sequential star-polymer synthesis. 


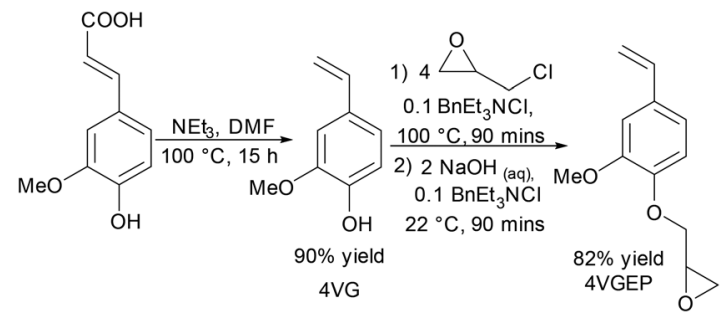

Scheme 1 Synthesis of 4VGEP from ferulic acid and epichlorohydrin.

a)
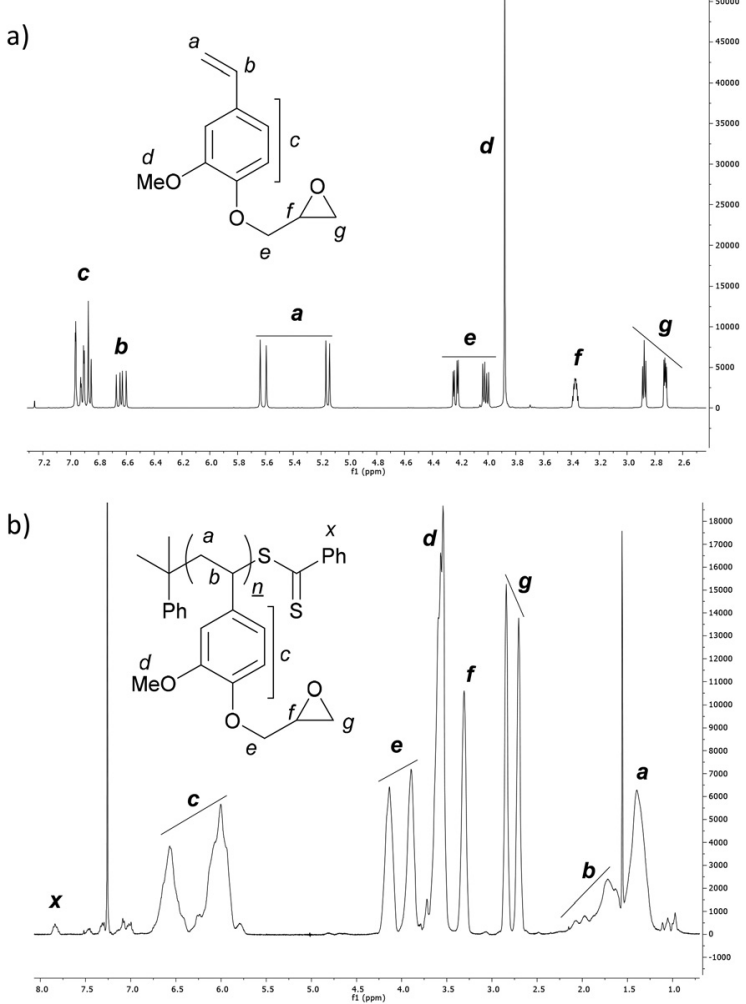

Fig. $1{ }^{1} \mathrm{H}$ NMR spectrum of (a) 4VGEP $\left(\mathrm{CDCl}_{3}, 25^{\circ} \mathrm{C}\right)$ and (b) P4VGEP $\left(\mathrm{CDCl}_{3}, 60^{\circ} \mathrm{C}\right)$.

Moreover, as ester linkages are hydrolytically sensitive, this could enable selective degradation. Such a strategy has been employed by $\mathrm{Wu}$ and coworkers with poly(propylene carbonate)-block-poly(4-vinylcatechol acetonide) in which the polycarbonate component was selectively degraded under mildly alkaline conditions to form membranes with monodisperse nanopores. ${ }^{28}$ Tsarevsky and coworkers have also reported on the atom-transfer-radical-polymerisation (ATRP) and RAFTpolymerisation of 4-vinylphenyl glycidyl ether derivatives to give linear and star-shaped epoxide-containing, but nondegradable, polyolefins. ${ }^{36,38}$ Using a dual-functional initiator strategy, Qiao and coworkers have combined ATRP of vinyl monomers with ROP of lactones for the synthesis of mixed poly(olefin/ester) star polymers with various architectures. ${ }^{39,40}$ The polyester component of these star polymers could be selectively hydrolysed, although scope for core or arm functionality would here be limited by the availability and polymerisability of (di)lactones used.

Presented here is the polymerisation of 4VGEP, a fully-bio derived, epoxide-functionalised, styrene-type monomer which is readily synthesised. ${ }^{41}$ Block copolymers with styrene diethylacrylamide (DEAA) have been used to synthesise star polymers, by an arm first approach, using a sequential, tandem living RAFT/ROCOP-based strategy. Controlled degradation of the styrene-based star polymer's core to linear polymers was achieved under acidic conditions.

\section{Results and discussion}

Decarboxylation of ferulic acid in the presence of triethylamine $\left(\mathrm{NEt}_{3}\right)$ afforded $4 \mathrm{VG}$ as previously reported (Scheme 1). ${ }^{22}$ 4 VGEP was synthesised in good yield by reaction of $4 \mathrm{VG}$ with epichlorohydrin (which may be derived from glycerol ${ }^{42}$ ) in the presence of the phase transfer catalyst, $\mathrm{BnEt}_{3} \mathrm{NCl}^{41}$

Recrystallisation from hexane afforded the monomer in sufficient purity for RAFT-polymerisation (Fig. 1a).

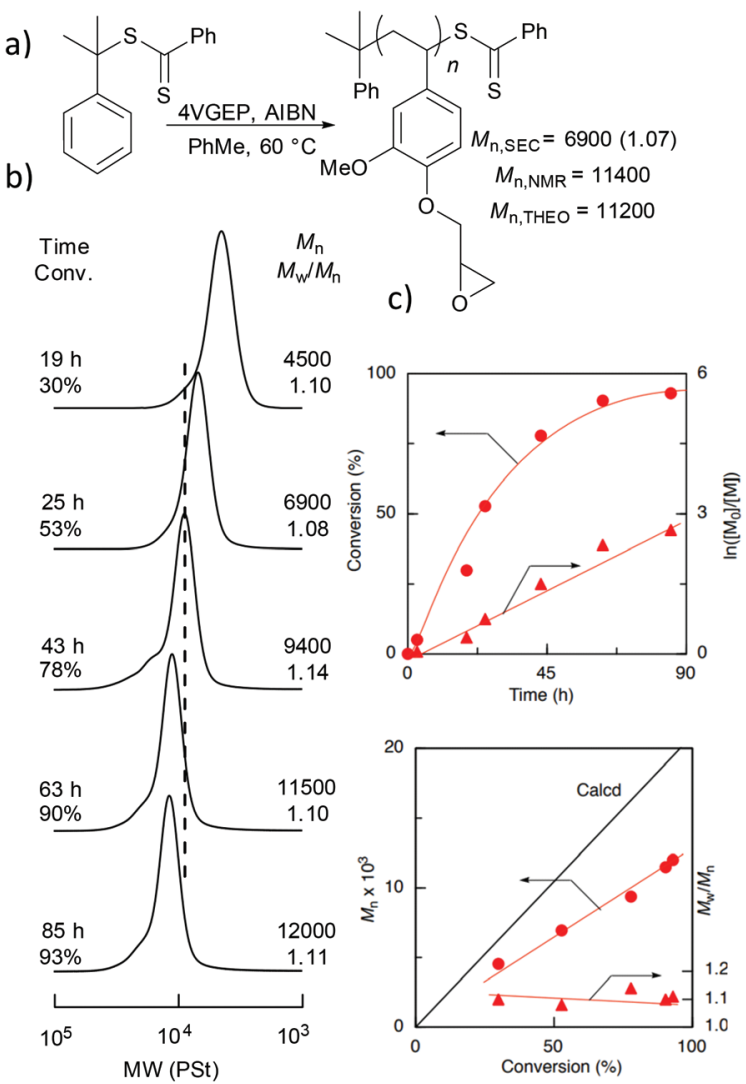

Fig. 2 (a) Homopolymerisation of 4VGEP at $60{ }^{\circ} \mathrm{C}$ at $[4 \mathrm{VGEP}]_{0}:[\mathrm{CDB}]_{0}:[\mathrm{AIBN}]_{0}$ loadings of $100: 1: 0.25$ in toluene. $[4 \mathrm{VGEP}]_{0}=$ $3.0 \mathrm{~mol} \mathrm{~L}^{-1}$ (b) associated SEC chromatograms (c) time vs. conversion and conversion vs. $M_{\mathrm{n}}$ and vs. $\Xi_{\mathrm{M}}$ plots. 
The homopolymerisation of 4VGEP was initiated by azobisisobutyronitrile (AIBN) in the presence of cumyl dithiobenzoate (CDB) at $60{ }^{\circ} \mathrm{C}$ (Fig. 2). The polymerisation demonstrated typical living characteristics, with good agreement between theoretical and observed molecular weights $\left(M_{\mathrm{n}, \mathrm{SEC}}\right)$ and narrow molecular weight distributions $\left(D_{\mathrm{M}}=1.08-1.14\right)$ as determined by size exclusion chromatography (SEC) (Fig. 2b and c). As expected, ${ }^{1} \mathrm{H}$ NMR spectroscopy showed that the epoxide functionality remained intact post-RAFT polymerisation (Fig. 1b).

Next, block copolymers of 4 VGEP and styrene (St- $b$-4VGEP), and 4VGEP and diethylacrylamide (DEAA, DEAA- $b$-4VGEP) were synthesised (Fig. 3). Styrene and DEAA were chosen as comonomers as they are known to form hydrophobic and hydrophilic polymers, respectively. First, polystyrene (PSt) and polyDEAA (PDEAA) were prepared and isolated as macro-RAFT agents, targeting a degree of polymerisation (DP) between 100-200. The polymerisations were performed at $[\text { monomer }]_{0}:[\mathrm{CDB}]_{0}$ ratios of $200: 1$. For styrene, the reaction was performed in bulk in the absence of initiator, whereas for DEAA, the polymerisation was carried out at $60^{\circ} \mathrm{C}$ using AIBN
$\left([\mathrm{DEAA}]_{0}:[\mathrm{AIBN}]_{0}:[\mathrm{CDB}]_{0}=200: 0.2: 1\right)$. The reactions were terminated once monomer conversion reached over $50 \%$.

With these macro-RAFT agents in hand, copolymerisations with 4VGEP were attempted, targeting the formation of block copolymers with a short 4VGEP segment at a styrene : 4VGEP and DEAA: 4VGEP ratio of approximately 10:1 (Fig. 3a). The initial reaction was carried out with the macro-RAFT PSt at loadings of $[4 \mathrm{VGEP}]_{0}:[\mathrm{AIBN}]_{0}:[\mathrm{PSt}]_{0}$ of $20: 0.2: 1$ at $60{ }^{\circ} \mathrm{C}$ in toluene. The large amount of toluene necessary to completely solubilise both 4 VGEP and PSt at $60^{\circ} \mathrm{C}$ resulted in [4VGEP $]_{0}$ of $0.75 \mathrm{~mol} \mathrm{~L}^{-1}$, which is relatively low for RAFT polymerization to be effective. Consequently, while the addition of short blocks of 4VGEP to the macro-RAFT PSt chains was observed by ${ }^{1} \mathrm{H}$ NMR spectroscopy at conversions of $19 \%$ and over, only slight increases in molecular weight and small shifts in the SEC curves were detected, with long reaction times resulting in small shoulders at higher molecular weight in the associated SEC chromatograms. These observations suggested a non-negligible occurrence of radical-radical termination reactions along with a slow polymerisation due to the low concentration of $4 \mathrm{VGEP}$, and indicated that the initial $[4 \mathrm{VGEP}]_{0}$ and tempera-



Fig. 3 (a) Synthesis of St- $b-4$ VGEP and DEAA- $b-4$ VEP. (b) SEC chromatograms for the block copolymerisation of PSt and 4VGEP at [PSt $]_{0}$ : [4VGEP] $]_{0}$ $50: 1$ and PDEAA and 4VGEP at [PDEAA $]_{0}:[4 \mathrm{VGEP}]_{0}$ of $100: 1$ at $110{ }^{\circ} \mathrm{C}$ in o-DCB. [4VGEP] $]_{0}=2.0 \mathrm{~mol} \mathrm{~L}^{-1}$. (c) ${ }^{1} \mathrm{H}$ NMR spectrum of St- $b-4 \mathrm{VGEP}$ $\left(\mathrm{CDCl}_{3}, 60^{\circ} \mathrm{C}\right)$ and $(\mathrm{d})$ DEAA- $b-4 \operatorname{VEP}\left(\mathrm{CDCl}_{3}, 60^{\circ} \mathrm{C}\right)$. 
ture were insufficient for the block copolymerisation to proceed as intended.

A second reaction was carried out at $110{ }^{\circ} \mathrm{C}$ in orthodichlorobenzene (o-DCB) in the absence of initiator at $[4 \mathrm{VGEP}]_{0}:[\mathrm{PSt}]_{0}$ of $50: 1$, giving a $[4 \mathrm{VGEP}]_{0}=2.0 \mathrm{~mol} \mathrm{~L}^{-1}$ (Fig. 3b). No initiator is necessary for RAFT polymerization of styrene and its derivatives at high temperature because thermal initiation generally occurs. ${ }^{22}$ Satisfyingly, the polymerisation proceeded well, with a significant increase in the copolymer $M_{\mathrm{n}, \mathrm{SEC}}$ and no shoulders detected in the SEC chromatograms. At 29\% conversion, the reaction was quenched to get a short block of 4 VGEP for the subsequent cross-linking reaction.

The conditions were then applied for the 4VGEP copolymerisation with PDEAA macro-RAFT agent, at loadings of $[4 \mathrm{VGEP}]_{0}:[\mathrm{PDEAA}]_{0}$ of $100: 1$, to maintain $[4 \mathrm{VGEP}]_{0}$ of $2.0 \mathrm{~mol} \mathrm{~L}^{-1}$. As expected, the polymerisation proceeded well as indicated by ${ }^{1} \mathrm{H}$ NMR spectroscopy and SEC analysis (Fig. 3b). The reaction was quenched at $9 \%$ conversion. For both macroRAFT agents, the epoxide remained intact following polymerisation (Fig. 3c and d).

Star polymer synthesis was then attempted using epoxide/ anhydride ring opening copolymerisation (ROCOP) (Fig. 4). A ROCOP strategy was pursued as it was postulated that the resulting polyester core would allow for the selective degradation of the star polymers.

cis-4-Cyclohexene-1,2-dicarboxylic anhydride (CDCA) was chosen as the co-monomer as it is well studied in ROCOP and the presence of a double bond could allow for further functionalisation of the star polymer if desired. In addition, the parent diacid of CDCA may be sustainably-derived from 1,4-cyclohexadiene ${ }^{43}$ or the anhydride may be synthesised directly via a Diels-Alder reaction of renewably-sourced 1,3butadiene $^{44}$ and maleic anhydride. ${ }^{45}$

a)


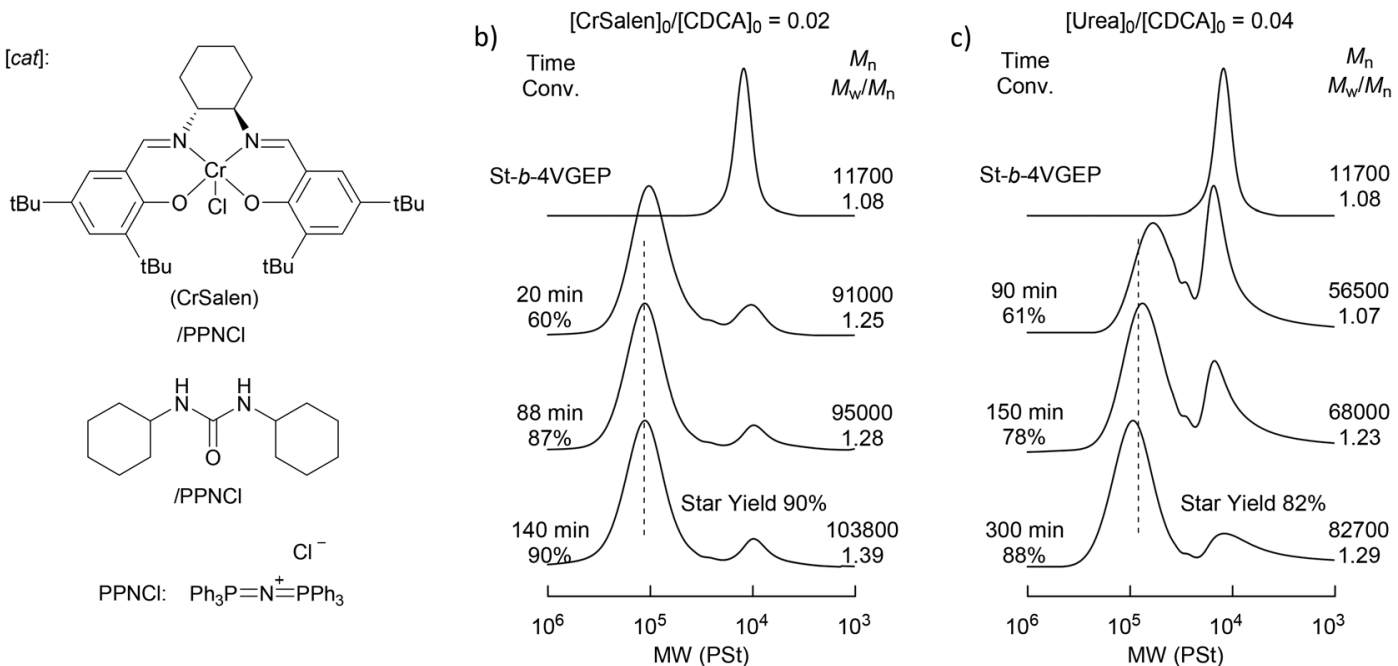

Fig. 4 (a) Synthesis of star polymers from St-b-4VGEP and CDCA. SEC chromatograms for the (b) CrSalen (2 mol\%) and (c) urea (4 mol\%) catalysed ROCOP of St- $b-4$ VGEP and CDCA. mol\% given with respect to anhydride loading. [St- $b-4 \mathrm{VGEP}]=0.01 \mathrm{~mol} \mathrm{~L}^{-1}$.

Table 1 Star polymer formation from St- $b-4$ VGEP and DEAA- $b-4$ VEP

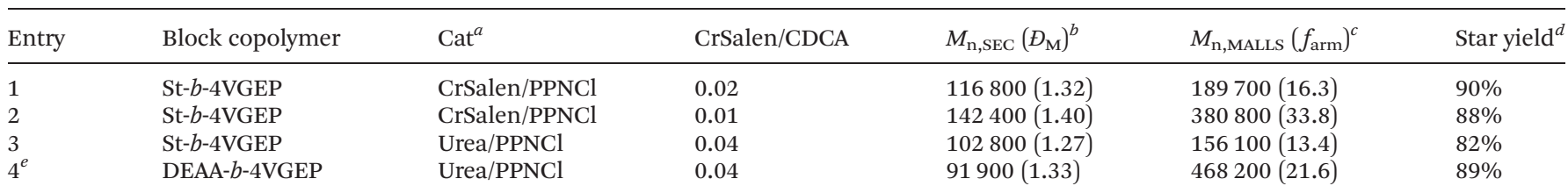

Reactions carried out at [block copolymer $]_{0}:[\mathrm{CDCA}]_{0}$ of $1: 14$ at $110{ }^{\circ} \mathrm{C}$ in $o$-DCB. [block copolymer] $=0.01$ mol L $\mathrm{L}^{-1}$ unless otherwise stated. ${ }^{a}$ Cat : PPNCl added in a $1: 1$ ratio. ${ }^{b}$ Determined against a set of PSt standards, $D_{\mathrm{M}}=M_{\mathrm{w}} / M_{\mathrm{n}} \cdot{ }^{c} f_{\mathrm{arm}}=\left(M_{\mathrm{n}, \mathrm{MALLS}}(\mathrm{Star})-14 \times \operatorname{conv} \times M_{\mathrm{w}}(\mathrm{CDCA})\right) /$ $M_{\mathrm{n}, \mathrm{MALLS}}(\mathrm{St}-b-4 \mathrm{VGEP}) .{ }^{d}$ Taken from relative area of associated SEC chromatogram peak. ${ }^{e}$ Reaction carried out at $[\mathrm{DEAA}-b-4 \mathrm{VGEP}]_{0}:[\mathrm{CDCA}]_{0}$ of $1: 16$. 
Reactions were initially carried out with the block copolymer St- $b$-4VGEP using a binary catalyst comprising a chromium salen chloride complex (CrSalen) and bis(triphenylphosphoranylidene)ammonium chloride salt (PPNCl). CrSalen was selected as it is a commercial and versatile ROCOP catalyst. $^{46,47}$ A $[\mathrm{St}-b-4 \mathrm{VGEP}]_{0}:[\mathrm{CDCA}]_{0}$ ratio of $1: 14$ was chosen based on the copolymerisation NMR data, in order to achieve equal anhydride and epoxide concentrations. ROCOP was found to proceed readily at loadings of [St- $b$ $4 \mathrm{VGEP}]_{0}:[\mathrm{CDCA}]_{0}:[\mathrm{CrSalen}]_{0}:[\mathrm{PPNCl}]_{0}$ of $10: 140: 2.8: 2.8$ and $10: 140: 1.4: 1.4$, with $[\mathrm{St}-b-4 \mathrm{VGEP}]_{0}=0.01 \mathrm{~mol} \mathrm{~L} \mathrm{~L}^{-1}$

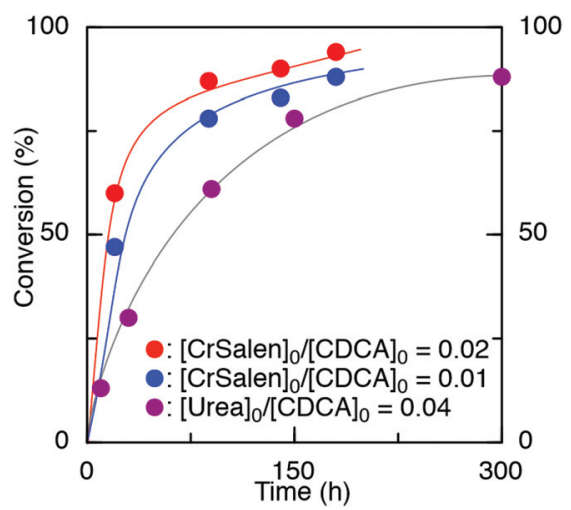

Fig. 5 Time vs. \% conversion for the CrSalen and urea catalysed ROCOP of St- $b$-4VGEP and CDCA at loadings of: (red) [St- $b$ 4 VGEP $]_{0}:[C D C A]_{0}:[\text { CrSalen }]_{0}:[P P N C l]_{0}=10: 140: 2.8: 2.8$, (blue) [St$b-4 \mathrm{VGEP}]_{0}:[\mathrm{CDCA}]_{0}:[\mathrm{CrSalen}]_{0}:[\mathrm{PPNCl}]_{0}=10: 140: 1.4: 1.4$ and (purple) $[\text { St- } b-4 \mathrm{VGEP}]_{0}:[\mathrm{CDCA}]_{0}:\left[\right.$ [urea $_{0}:[\mathrm{PPNCl}]_{0}=10: 140: 5.6: 5.6$. $\mathrm{mol} \%=[\mathrm{CrSalen}]_{0} /[\mathrm{CDCA}]_{0} .[\mathrm{St}-b-4 \mathrm{VGEP}]_{0}=0.01 \mathrm{~mol} \mathrm{~L}^{-1}$.

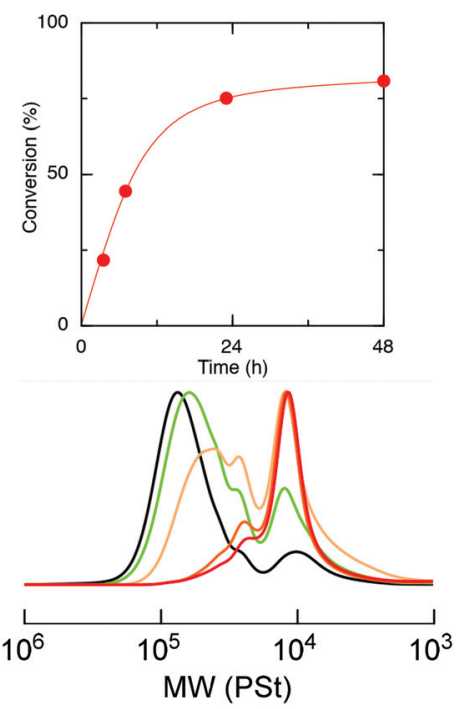

Fig. 6 (Top) conversion of star-St- $b$-4VGEP to hydrolysis products vs. time. Conversions were calculated by the relative integration of the SEC traces of the star-St-b-4VGEP to linear polymer by RI detection. (Bottom) RI vs. $M_{\mathrm{n}}$ (PSt) for the acid hydrolysis of star-St- $b$-4VGEP at $100{ }^{\circ} \mathrm{C}$ (black $=$ crude star polymer, green $=3.5 \mathrm{~h}$ aliquot, orange $=7 \mathrm{~h}$ aliquot, deep orange $=23 \mathrm{~h}$ aliquot and red $=48 \mathrm{~h}$ aliquot).
(Table 1, entries 1 and 2; Fig. 5). Good control was exhibited in the ROCOP, with narrow $\bigoplus_{\mathrm{M}}(1.32-1.35)$ obtained, and absolute molecular weight approximately doubling upon decreasing the CrSalen loading from $2 \mathrm{~mol} \%\left(M_{\mathrm{n}, \mathrm{MALLS}}=189700 \mathrm{~g} \mathrm{~mol}^{-1}\right)$ to $1 \mathrm{~mol} \%\left(M_{\mathrm{n}, \mathrm{MALLS}}=380800 \mathrm{~g} \mathrm{~mol}{ }^{-1}\right)$. Under otherwise analogous conditions, with $[\mathrm{St}-b-4 \mathrm{VGEP}]_{0}$ of $0.38 \mathrm{~mol} \mathrm{~L}^{-1}$, insoluble residues were formed, likely as a result of star-star coupling.

Further study was carried out using a urea/PPNCl catalyst (Table 1 , entry 3$).{ }^{48}$ Here, a ratio of $[\text { urea }]_{0}:[\mathrm{CDCA}]_{0}$ of $1: 25$ was chosen as studies have shown that the urea catalyst is less active than the CrSalen. ${ }^{46}$ Again, the reaction was found to proceed readily at $[\mathrm{St}-b-4 \mathrm{VGEP}]_{0}:[\mathrm{CDCA}]_{0}:[\mathrm{urea}]_{0}:[\mathrm{PPNCl}]_{0}$ loadings of $10: 140: 5.6: 5.6$, albeit at a slower rate and with a slightly lower yield of star polymer obtained (Fig. 5). As expected from the higher catalyst loadings, absolute molecular weight was lower than for CrSalen $\left(M_{\mathrm{n}, \text { MALLS }}=156100 \mathrm{~g}\right.$ $\mathrm{mol}^{-1}$ ) while $\bigoplus_{\mathrm{M}}$ remained similar (1.27).

Given the low toxicity of dicyclohexyl urea and with potential biomedical applications in mind, the metal-free urea/ PPNCl catalyst was chosen to carry out further study with the DEAA- $b$-4VGEP copolymer. The reaction proceeded as expected at $[\mathrm{DEAA}-b-4 \mathrm{VGEP}]_{0}:[\mathrm{CDCA}]_{0}:[\mathrm{urea}]_{0}:[\mathrm{PPNCl}]_{0}$ loadings of $10: 170: 6.8: 6.8$ (Table 1 , entry 4 , Fig. S5†). Good control was sustained in the ROCOP $\left(\bigoplus_{\mathrm{M}}\right.$ 1.33) with high absolute molecular weights $\left(M_{\mathrm{n}, \mathrm{MALLS}} 468200 \mathrm{~g} \mathrm{~mol}^{-1}\right.$ ) obtained, demonstrating the versatility of the urea/PPNCL catalyst.

Selective degradation of the ester-linked core of the star polymer, star-St- $b$-4VGEP, was then attempted. We hypothesised that the ester-links would undergo hydrolysis whereas the vinyl polymers arms would remain intact, enabling controlled degradation of the core. ${ }^{39}$ Hydrolysis was attempted under acidic conditions using a 1:4 trifluoroacetic acid

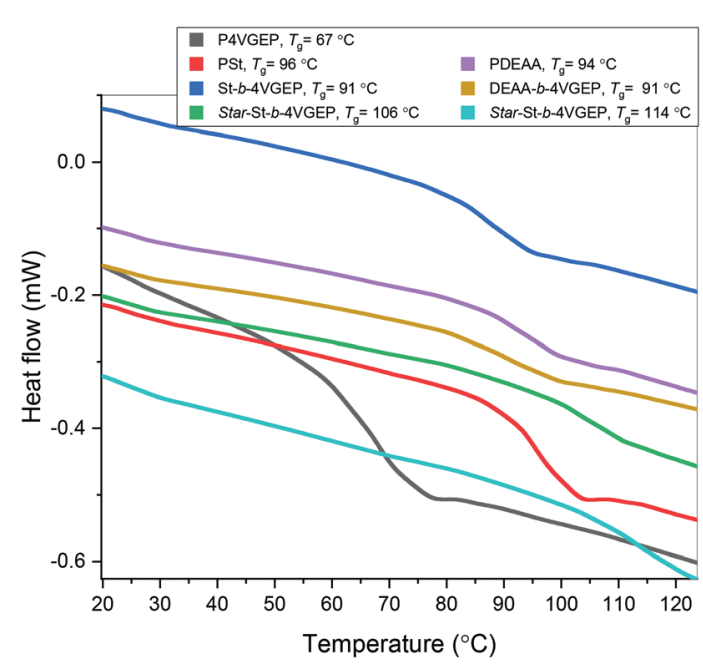

Fig. 7 DSC thermograms of all polymers isolated in this study: P4VGEP (dark grey, $M_{\mathrm{n}, \mathrm{SEC}}=6900$ ), PSt (red, $\left.M_{\mathrm{n}, \mathrm{SEC}}=9800\right)$, St- $b$-4VGEP (blue, $\left.M_{n, S E C}=11700\right)$, star-St- $b-4$ VGEP (green, $\left.M_{n, S E C}=116800\right)$, PDEAA (lilac, $\left.M_{\mathrm{n}, \mathrm{SEC}}=13900\right)$, DEAA- $b-4 \mathrm{VGEP}\left(\right.$ gold, $\left.M_{\mathrm{n}, \mathrm{SEC}}=18500\right)$ and starSt- $b$-4VGEP (cyan, $\left.M_{n, \sec }=91900\right)$. 
(TFA) : $\mathrm{H}_{2} \mathrm{O}$ mixture. At room temperature, no reactivity was observed in a DCM solution of the star, up to $120 \mathrm{~h}$, as confirmed by SEC analysis. However, satisfyingly, at $100{ }^{\circ} \mathrm{C}$ in toluene, near complete degradation of the core was possible after $48 \mathrm{~h}$ to form a polymer of $M_{\mathrm{n}, \mathrm{SEC}}\left(\bigoplus_{\mathrm{M}}\right)=11200$ (1.38) (Fig. 6 and S14†).

Thermogravimetric analysis (TGA) showed that all novelpolymers had a temperature of $5 \%$ mass loss $\left(T_{\mathrm{d} 5}\right)$ of over $300{ }^{\circ} \mathrm{C}$. The formation of the star polymers resulted in a slight decrease in $T_{\mathrm{d} 5}$ which may be attributed to the incorporation of the thermally less-stable $\mathrm{C}-\mathrm{O}$ bonds, formed as part of the ester linkages in the polymeric stars. Similarly, the homopolymer, P4VGEP, has a lower $T_{\mathrm{d} 5}$ than both the block copolymers, St- $b$-4VGEP and DEAA- $b$-4VGEP, due to the greater density of epoxide-functionalised pendant arms (Fig. S13 $\dagger$ ).

Differential scanning calorimetry (DSC) indicated that all synthesised polymers were amorphous with $T_{\mathrm{g}}$ 's ranging from 67-114 ${ }^{\circ} \mathrm{C}$ (Fig. 7). No microphase separation was observed between the styrene and 4VGEP or the DEAA and 4VGEP blocks copolymers. For both polymers, incorporation of the 4VGEP block resulted in a slight decrease in the polymers' $T_{\mathrm{g}}$ (St- $b$-4VGEP $-5{ }^{\circ} \mathrm{C}$, DEAA- $b$ - 4 VGEP $-3{ }^{\circ} \mathrm{C}$ ). Formation of the star polymers resulted in greater increase in $T_{\mathrm{g}}$, consistent with the incorporation of a rigid cyclohexyl unit in the polymer (star $\mathrm{St}-b$-4VGEP $+15^{\circ} \mathrm{C}$, star DEAA- $b-4$ VGEP $\left.=+23^{\circ} \mathrm{C}\right)$.

\section{Conclusions}

In conclusion, a novel epoxide functionalised styrene-type polymer has been synthesised from a ferulic acid and a glycerol derivative. Synthesis of PSt and PDEAA macro-RAFT agents followed by copolymerisation with 4VGEP formed the block copolymers, St- $b$-4VGEP and DEAA- $b$-4VGEP. Star polymer synthesis was then attempted by epoxide/anhydride ROCOP using a CrSalen/PPNCl and a urea/PPNCl catalyst. Initial study with St- $b$-4VGEP showed the ROCOP proceeded readily for both systems. The urea catalyst was then applied in the star polymer synthesis using the block copolymer, DEAA- $b$ 4 VEP, forming the star polymer, star DEAA- $b$-4VEGP in good yield. Finally, controlled degradation of the star polymer, star St-b4VGEP, was demonstrated under acidic conditions at $100{ }^{\circ} \mathrm{C}$. All novel polymers were fully characterized by TGA and DSC. Future studies will focus on using 4VGEP to synthesise polymers with dynamic cross-links to enable the formation of materials with self-healing properties.

\section{Conflicts of interest}

There are no conflicts to declare.

\section{Acknowledgements}

This work was supported by the Japan Society for the Promotion of Science (JSPS) Summer program fellowship
(SP19105) granted to T. M. M. A. B. acknowledges the Royal Society (UF/160021 fellowship) for research funding.

\section{References}

1 Y. Zhu, C. Romain and C. K. Williams, Nature, 2016, 540, 354-362.

2 A. J. Ragauskas, G. T. Beckham, M. J. Biddy, R. Chandra, F. Chen, M. F. Davis, B. H. Davison, R. A. Dixon, P. Gilna, M. Keller, P. Langan, A. K. Naskar, J. N. Saddler, T. J. Tschaplinski, G. A. Tuskan and C. E. Wyman, Science, 2014, 344, 124684.

3 D. K. Schneiderman and M. A. Hillmyer, Macromolecules, 2017, 50, 3733-3749.

4 G. L. Gregory, E. M. Lopez-Vidal and A. Buchard, Chem. Commun., 2017, 53, 2198-2217.

5 V. Delplace and J. Nicolas, Nat. Chem., 2015, 7, 771-784.

6 J. M. Ren, T. G. McKenzie, Q. Fu, E. H. H. Wong, J. Xu, Z. An, S. Shanmugam, T. P. Davis, C. Boyer and G. G. Qiao, Chem. Rev., 2016, 116, 6743-6836.

7 Y. Wang and S. M. Grayson, Adv. Drug Delivery Rev., 2012, 64, 852-865.

8 C. Yang, S. Q. Liu, S. Venkataraman, S. J. Gao, X. Ke, X. T. Chia, J. L. Hedrick and Y. Y. Yang, J. Controlled Release, 2015, 208, 93-105.

9 Y. K. Chong, I. Zainol, C. H. Ng and I. H. Ooi, J. Polym. Res., 2019, 26, 79.

10 T. K. Georgiou, Polym. Int., 2014, 63, 1130-1133.

11 Y. Wang, C.-Y. Hong and C.-Y. Pan, Biomacromolecules, 2012, 13, 2585-2593.

12 K.-I. Fukukawa, R. Rossin, A. Hagooly, E. D. Pressly, J. N. Hunt, B. W. Messmore, K. L. Wooley, M. J. Welch and C. J. Hawker, Biomacromolecules, 2008, 9, 1329-1339.

13 T. R. Bagby, S. Duan, S. Cai, Q. Yang, S. Thati, C. Berkland, D. J. Aires and M. Laird Forrest, Eur. J. Pharm. Sci., 2012, 47, 287-294.

14 V. Rodionov, H. Gao, S. Scroggins, D. A. Unruh, A.-J. Avestro and J. M. J. Fréchet, J. Am. Chem. Soc., 2010, 132, 25702572.

15 T. J. Dickerson, N. N. Reed and K. D. Janda, Chem. Rev., 2002, 102, 3325-3344.

16 C. Boyer, M. H. Stenzel and T. P. Davis, J. Polym. Sci., Polym. Chem., 2011, 49, 551-595.

17 G. Moad, E. Rizzardo and S. H. Thang, Aust. J. Chem., 2012, 65, 985-1076.

18 J. Ferreira, J. Syrett, M. Whittaker, D. Haddleton, T. P. Davis and C. Boyer, Polym. Chem., 2011, 2, 1671-1677.

19 Q. Qiu, G. Liu and Z. An, Chem. Commun., 2011, 47, 1268512687.

20 M. Uchiyama, K. Satoh, T. G. McKenzie, Q. Fu, G. G. Qiao and M. Kamigaito, Polym. Chem., 2017, 8, 59725981.

21 N. Usuki, H. Okura, K. Satoh and M. Kamigaito, J. Polym. Sci., Polym. Chem., 2018, 56, 1123-1127. 
22 H. Takeshima, K. Satoh and M. Kamigaito, Macromolecules, 2017, 50, 4206-4216.

23 H. Takeshima, K. Satoh and M. Kamigaito, Polym. Chem., 2019, 10, 1192-1201.

24 H. Takeshima, K. Satoh and M. Kamigaito, Polymers, 2018, 10, 1404.

25 W. S. J. Li, V. Ladmiral, H. Takeshima, K. Satoh, M. Kamigaito, M. Semsarilar, C. Negrell, P. LacroixDesmazes and S. Caillol, Polym. Chem., 2019, 10, 31163126.

26 S. Paul, Y. Zhu, C. Romain, R. Brooks, P. K. Saini and C. K. Williams, Chem. Commun., 2015, 51, 6459-6479.

27 Y.-Y. Zhang, G.-W. Yang and G.-P. Wu, Macromolecules, 2018, 51, 3640-3646.

28 H.-J. Zhou, G.-W. Yang, Y.-Y. Zhang, Z.-K. Xu and G.-P. Wu, ACS Nano, 2018, 12, 11471-11480.

29 Y. Wang, Y. Zhao, Y. Ye, H. Peng, X. Zhou, X. Xie, X. Wang and F. Wang, Angew. Chem., Int. Ed., 2018, 57, 3593-3597.

30 Y. Zhao, Y. Wang, X. Zhou, Z. Xue, X. Wang, X. Xie and R. Poli, Angew. Chem., Int. Ed., 2019, 58, 14311-14318.

31 A. Denk, S. Kernbichl, A. Schaffer, M. Kränzlein, T. Pehl and B. Rieger, ACS Macro Lett., 2020, 9, 571-575.

32 Y. Wang, Y. Zhao, S. Zhu, X. Zhou, J. Xu, X. Xie and R. Poli, Angew. Chem., 2020, 59, 5988-5994.

33 S. Zhu, Y. Zhao, M. Ni, J. Xu, X. Zhou, Y. Liao, Y. Wang and X. Xie, ACS Macro Lett., 2020, 9, 204-209.

34 Y.-Y. Zhang, G.-P. Wu and D. J. Darensbourg, Trends Chem., 2020, 2, 750-763.
35 D. C. McLeod and N. V. Tsarevsky, J. Polym. Sci., Polym. Chem., 2016, 54, 1132-1144.

36 D. C. McLeod and N. V. Tsarevsky, Polym. Int., 2014, 63, 868-875.

37 S. R. Woodruff, B. J. Davis and N. V. Tsarevsky, Macromol. Rapid Commun., 2014, 35, 186-192.

38 D. C. McLeod and N. V. Tsarevsky, Macromolecules, 2016, 49, 1135-1142.

39 J. T. Wiltshire and G. G. Qiao, Macromolecules, 2006, 39, 9018-9027.

40 J. T. Wiltshire and G. G. Qiao, J. Polym. Sci., Polym. Chem., 2009, 47, 1485-1498.

41 E. Zago, E. Dubreucq, J. Lecomte, P. Villeneuve, F. Fine, H. Fulcrand and C. Aouf, New J. Chem., 2016, 40, 77017710.

42 R. A. Sheldon, Green Chem., 2014, 16, 950-963.

43 M. Winkler, C. Romain, M. A. R. Meier and C. K. Williams, Green Chem., 2015, 17, 300-306.

44 C. Angelici, B. M. Weckhuysen and P. C. A. Bruijnincx, ChemSusChem, 2013, 6, 1595-1614.

45 H. K. Hall, P. Nogues, J. W. Rhoades, R. C. Sentman and M. Detar, J. Org. Chem., 1982, 47, 1451-1455.

46 A. M. DiCiccio and G. W. Coates, J. Am. Chem. Soc., 2011, 133, 10724-10727.

47 T. Stosser, G. S. Sulley, G. L. Gregory and C. K. Williams, Nat. Commun., 2019, 10, 9.

48 L. Lin, J. Liang, Y. Xu, S. Wang, M. Xiao, L. Sun and Y. Meng, Green Chem., 2019, 21, 2469-2477. 